INVESTIGACIÓN EDUCATIVA

\title{
Percepciones estudiantiles del uso de modelos 3D en la enseñanza de la microscopía en veterinaria
}

\author{
Rizzo $D^{1}$, Borlido $C^{2}$, Passarini $\mathrm{J}^{2^{*}}$, Pedrana $\mathrm{G}^{1}$ \\ ${ }^{1}$ Departamento de Biociencias, Facultad de Veterinaria, Universidad de la República (UdelaR). \\ ${ }^{2}$ Departamento de Educación Veterinaria, Facultad de Veterinaria, UdelaR. \\ * Correspondencia: José Pasarini. Facultad de Veterinaria, UdelaR. Alberto Lasplaces 1550, 11600 \\ Montevideo, Departamento de Montevideo, Uruguay. E-mail: josepasa@gmail.com
}

Recibido: 6 Julio 2020. Aceptado: 24 de Agosto 2020. Disponible en línea: 2 Febrero 2021

Editor: R. Sobrero

RESUMEN. Mundialmente la impresión 3D ha impactado en la enseñanza de las ciencias. Sin embargo, es poco analizada su utilización en las aulas universitarias desde la perspectiva del estudiante. Es por esto que nos planteamos analizar las percepciones estudiantiles sobre el uso de modelos 3D en cursos de primer año en la Facultad de Veterinaria. Para ello se realizó un cuestionario en línea en el Entorno Virtual de Aprendizaje y en el Instagram de Histología. Los resultados indican una percepción positiva y un interés por el uso de modelos 3D. Asimismo, se realizaron numerosas y variadas sugerencias por parte de los estudiantes de generar nuevos modelos para mejorar la comprensión de ciertos temas. Por lo tanto, los resultados indican que el trabajo de aula utilizando modelos 3D acerca al estudiante a representaciones tridimensionales de imágenes microscópicas. Las actividades con modelos 3D son consideradas por los estudiantes como facilitadores de la comprensión de los temas. El uso de este recurso didáctico promueve el desarrollo de estrategias cognitivas en los estudiantes, como ser la extrapolación de imágenes microscópicas a su representación tridimensional. Se sugiere combinar saberes tecnológicos y didácticos para apoyar a docentes y estudiantes para la generación de dichos modelos.

\begin{abstract}
SUMMARY. Student perceptions of the use of 3D models in microscopy veterinary education. 3D printing has impacted science education all over the world. However, the analysis at university classrooms from the student's perspective is scarce. Consequently, we aim to analyze student perceptions on the use of 3D models in first-year courses at the Veterinary Faculty. We performed an online questionnaire at the Virtual Learning Environment and on the Instagram of Histology account. The results indicate a positive perception and an interest in the use of 3D models. In addition, many and interesting suggestions were made by students to generate new models, as their declare that they improve understanding of certain topics. Therefore, the results indicate that working with didactic methodologies as the use of technological resources 3D models at classroom brings the student closer to their threedimensional representations of microscopic images. The planned activities using 3D models are considered by the students as facilitators of the understanding of the topics. The use of these didactic resource promotes the development of cognitive strategies in students, such as the extrapolation of microscopic images to their three-dimensional representation. We suggested to combine technological and didactic knowledge to support teachers and students for the generation of such models.
\end{abstract}

Palabras clave: modelos 3D, enseñanza universitaria, aprendizaje, biología, histología, veterinaria

Keywords: three dimensional technologies, learning, histology, teaching, veterinary

\section{Introducción}

La inclusión de Tecnologías de la Información y de la Comunicación (TIC) en la educación superior ha sido creciente, cambiando la enseñanza y los aprendizajes. Las TIC aparecen como un recurso innovador que influyen en aspectos socioculturales y permiten una interacción diferente con el contexto a través de nuevas metodologías de enseñanza (Duque et al., 2012).

Un aspecto fundamental de la creciente incorporación de TIC es su impacto, tanto en la enseñanza como en el aprendizaje, por estímulo motivacional generado en los estudiantes. La motivación para el aprendizaje y se ha observado que disminuye cuando los estudiantes pasan de la educación primaria a la secundaria (Castro et al., 2010) y particularmente parece ser menor en contenidos vinculados a ciencia y tecnología (Vázquez y Manassero, 2008).

Existen diversas herramientas asociadas a las TIC, desde el uso de plataformas educativas a distancia hasta aquellas que incorporan materiales didácticos para las actividades de enseñanza presenciales.

El estudio se centró en la incorporación de modelos 3D en un curso de la carrera de veterinaria de Uruguay. Es necesario aclarar que, en este caso, se refiere a la experiencia con impresiones 3D de piezas de estudio, elaboradas por impresoras 3D de uso doméstico 
capaces de realizar réplicas tridimensionales a partir de un diseño específico o un escaneo previo de los objetos a copiar (Pérez-Mañanes et al., 2016). Esto se diferencia del uso de la virtualidad inmersiva que genera imágenes tridimensionales que dan sensación de estar dentro de un ambiente determinado (Mejía et al., 2019).

Entre los usos de los modelos impresos en 3D en el área de la salud humana, está la fabricación de órganos o tejidos (Adrián César-Juárez et al., 2018), para prácticas y ensayos previos a las intervenciones en humanos, como, por ejemplo, sucede en las cirugías faciales en Uruguay (Mantrana et al., 2018; Vallarta et al., 2011) o en intervenciones cardiológicas en Argentina (Juaneda et al., 2018), entre tantos ejemplos que se incrementan de forma sostenida en la región.

Por lo tanto, no sorprende que las universidades en condiciones de innovar comiencen a incorporar esta tecnología para que sus estudiantes adquieran habilidades que aporten de forma relevante al aprendizaje de la profesión en la cual buscan formarse (González Bello, 2018).

Actualmente, se puede considerar que la impresión 3D es una de las tecnologías indispensables en la enseñanza de las ciencias (Freeman et al., 2017). Los modelos 3D representan un recurso didáctico utilizado con una finalidad educativa para facilitar el desarrollo de las actividades formativas (Guerrero Armas, 2009). Existen múltiples ventajas pedagógicas dadas por el uso y creación de modelos 3D, como estimular el pensamiento creativo y la resolución de problemas a través del desarrollo de productos tangibles. Recientemente se han realizado estudios que demuestran las ventajas de la aplicación de modelos 3D en la enseñanza de medicina veterinaria en la Universidad de Barcelona (Suñol et al., 2019). Estudios previos en Australia evaluaron el impacto de modelos 3D en formato digital y no digital (físico) en anatomía, en estudiantes de medicina y odontología en las habilidades de los estudiantes de visualización espacial de las relaciones anatómicas comparando varios países del mundo (Azer y Azer, 2016).

En este sentido, en la Facultad de Veterinaria de la Universidad de la República (Uruguay) se realizó en el año 2018 un curso de posgrado Modelado e impresión $3 D$, en base a reportes previos en la Universidad de Groninger, Holanda. El uso de a partir de un atlas histológico con modelos 3D en línea con modelos de diversos tipos de epitelios, en la enseñanza en medicina humana en la Universidad de Dundee, mostró un impacto positivo en la percepción de los estudiantes (Sieben et al., 2017).

En este sentido, durante el año 2019 en Uruguay, en la Facultad de Veterinaria, en los cursos curriculares de Biología Celular y Molecular (BMC) en las actividades prácticas del módulo de Citología en el primer semestre y en el curso de Histología y Biología del Desarrollo en el segundo semestre, se realizaron las primeras experiencias de uso de modelos 3D histológicos como recurso didáctico. Desde el punto de vista docente la utilización de herramientas 3D es considerada un aporte a la comprensión de los temas histológicos. Sin embargo, consideramos necesario contar con la opinión de los estudiantes sobre el uso de estos modelos en el aula. Por lo tanto, el objetivo del presente estudio fue evaluar la percepción de los estudiantes sobre el uso de los modelos 3D en las actividades de enseñanza de células, tejidos y órganos a nivel microscópico.

\section{Materiales y métodos}

Se llevó a cabo un cuestionario en línea sobre el uso de los modelos 3D en el aula mediante la creación de un cuestionario en la plataforma Google Forms. El cuestionario se realizó al finalizar el segundo semestre del año 2019, al culminar el curso de Histología, con 506 estudiantes inscriptos. La encuesta constó de cuatro preguntas dicotómicas y seis de respuesta abierta (Caja 1). Las primeras dos preguntas fueron sobre el acceso a los modelos en ambos cursos. Las siguientes fueron referidas a los modelos observados en las clases prácticas. Por último, las preguntas fueron relacionadas con la percepción de los estudiantes sobre la mejora de la comprensión de temas histológicos a partir de dichos modelos (¿cuáles fueron de mayor utilidad?, ¿cuáles son los temas más complejos de comprender?). Para acceder al cuestionario en línea se generó un vínculo en la plataforma del Entorno Virtual de Aprendizaje (EVA) y en el Instagram de Histología. Los resultados obtenidos fueron analizados determinando los porcentajes de respuesta para cada pregunta dicotómica. Las preguntas abiertas fueron categorizadas según las sugerencias de creación de modelos realizadas por los estudiantes. La encuesta fue realizada por 140 estudiantes que representan el $28 \%$ de los estudiantes del curso de Histología en el año 2019.

\section{Resultados}

Los resultados indican que los estudiantes encuestados perciben el trabajo en sala con modelos 3D de Citología en $\mathrm{BMC}$, mientras que se registró un aumento $(59 \%$ citología versus $88 \%$ Histología) de la percepción de los modelos utilizados en el segundo semestre en Histología (Figura 1).

En cuanto a los modelos que fueron observados por los estudiantes en Citología y en Histología manifestaron que observaron modelos de vellosidades intestinales, cóclea y de glándula mamaria variando las percepciones en porcentajes entre cada curso (Figura 2).

Con respecto a si mejoró la comprensión de los temas a partir de los modelos 3D, los resultados indicaron que la mayoría de los estudiantes en Citología e Histología (77 y $71 \%$ respectivamente) consideran que el uso de modelos mejora la comprensión de los temas (Figura 3).

En relación con las preguntas vinculadas a los temas 
Caja 1. Encuesta sobre Usos de Modelos 3D en el aula.

1. ¿Ha tenido acceso a los modelos $3 D$ en clases de Histología?

- $\mathrm{si}$

- No

2. ¿Ha tenido acceso a los modelos $3 \mathrm{D}$ en clases de Citología?

- $\mathrm{Si}$

- No

3. ¿Los modelos 3D observados le han sido de ayuda para comprender los diferentes temas de citología? - sí

- no

4. En histología, los modelos 3D observados cंle sirvieron para comprender los temas dados en histología?

- si

- no

5. ¿Cuáles de los modelos 3D vio en clases de Histología?

6. Los modelos 3D ¿Le pareció útil para la comprensión de los temas a estudiar?

7. ¿Cuáles de los modelos 3D que se utilizaron en clase le resultó de más ayuda para la comprensión de los temas?

8. ¿Cuáles son los temas más complejos de comprender con los cuáles le gustaría tener un modelo 3D?

9. ¿Qué modelo 3D piensa que le ayudaría a mejorar la comprensión de los temas histológicos? Especifique célula, tejido u órgano.

¿Cómo piensa que podría mejorar la comprensión de ciertos temas de Histología?

considerados por los estudiantes como más complejos y si los modelos podrían mejorar su comprensión, se determinaron los temas más frecuentemente mencionados. En primer lugar, un $37 \%$ de los estudiantes manifestó querer utilizar modelos 3D del sistema urinario. En segundo lugar, un $28 \%$ manifestó interés sobre modelos de globo ocular, seguidos un $23 \%$ por temas de tejido nervioso y un $12 \%$ sobre el tema de órganos linfoideos (Figura 4).

Del mismo modo, los encuestados indicaron su interés para generar diversos tipos de modelos de sistema urinario, tejido linfoideo, ocular, sistema vascular, endócrino, digestivo y distintos tipos celulares (Tabla 1).

\section{Discusión}

En el presente estudio se indagaron las percepciones estudiantiles sobre el uso en el aula de los modelos 3D en biología, específicamente en cursos vinculados a microscopía de la célula, tejidos, órganos y sistemas.

En el ámbito científico, en para los profesores de ciencias y para los estudiantes, los modelos 3D representan herramientas clave tal como lo indican investigaciones de aula sobre el uso de modelos y analogías dentro de la pedagogía de la educación científica (Garas et al., 2018). Estudios previos sugieren que el reflexionar y debatir sobre la comprensión de los conceptos científicos, así como construir y criticar sus propios modelos resultan ser técnicas pedagógicas muy efectivas (Coll et al., 2005).

\section{a} Uso de modelos 3D en Citología -
Facultad Veterinaria 2019

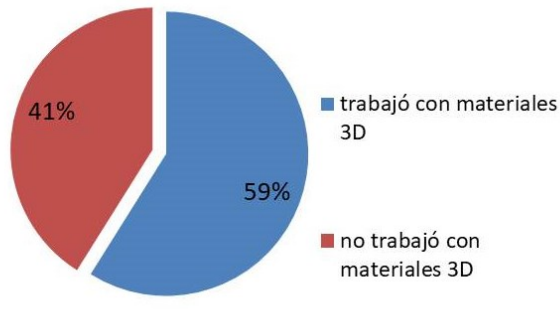

Uso de los modelos 3D en Histología

b Facultad Veterinaria 2019

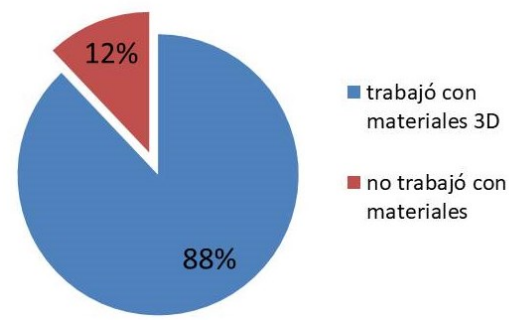

Figura 1. Uso de los modelos 3D en Citología en el curso de Biología Molecular y Celular (BMC) (a) y en Histología (b).

\section{a}

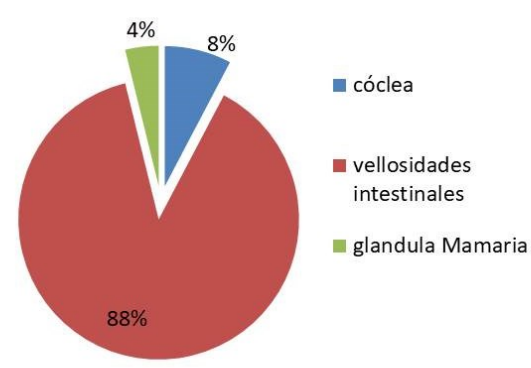

b

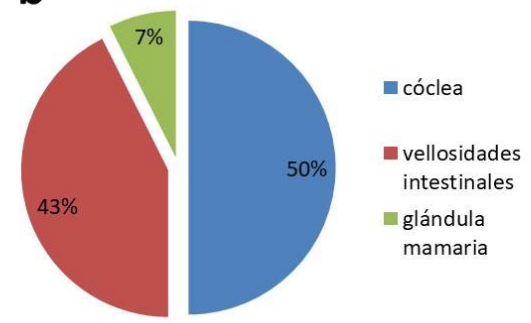

Figura 2. Respuestas de estudiantes sobre los modelos 3D observados en clase, en Citología (a) e Histología (b). 


\section{a Comprensión de temas en Citología}

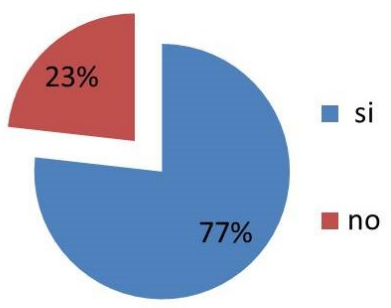

\section{b Comprensión de temas en Histología}

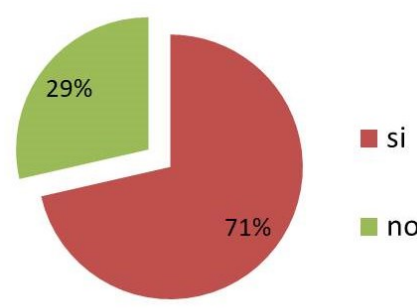

Figura 3. Percepción de los estudiantes sobre la mejora en la comprensión de los contenidos temáticos en Citología (a) y en Histología (b).

\section{a}

¿Cuáles son los temas más complejos de comprendery de los cuáles deberían haber modelos 3D?

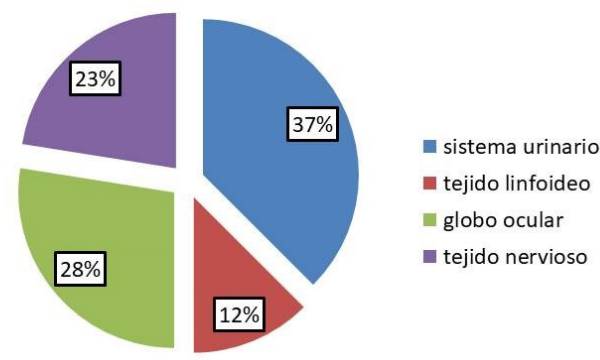

b

¿Qué modelo 3D piensa que le ayudaría a mejorar la

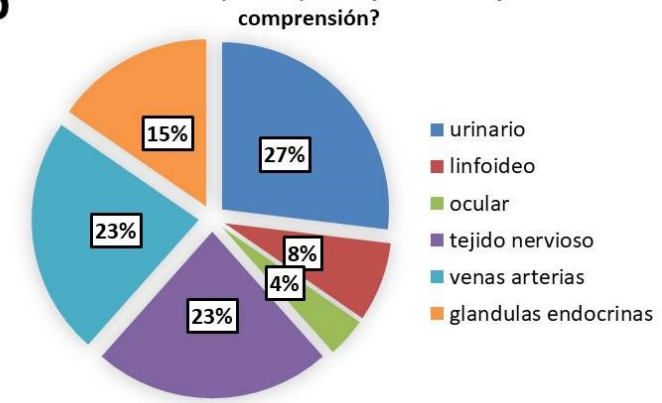

Figura 4. Gráfico de percepciones sobre la complejidad de temas y posibles modelos 3D a generar para mejorar la comprensión.

Según Moreira (2017), los modelos son constructos humanos $y$, por consiguiente, su existencia se debe a un proceso mental. Sin embargo, el acceso a los modelos mentales sería imposible, por lo que se requieren modelos expresados por un individuo en el dominio público a través de alguna forma de expresión (Gilbert, 2011). Por lo tanto, a través del uso didáctico de modelos $3 \mathrm{D}$ en el aula se posibilita que puedan expresarse modelos mentales que de otro modo serían complejos de determinar.

El potenciar el uso de los modelos 3D como un recurso didáctico facilita el desarrollo de estrategias cognitivas en los estudiantes. Estos modelos, usados por los docentes en las aulas permitió que los estudiantes extrapolaran a partir de imágenes capturadas de los microscopios en un plano y la confrontaran con su representación tridimensional. Tal como mencionan estudios previos, las visualizaciones nos permiten percibir y pensar sobre las relaciones entre los elementos que serían difícil de comprender sin ellas (Uttal y Doherty, 2008).

Tabla 1. Respuestas a la pregunta: '¿Qué modelo 3D piensas que te ayudaría a mejorar la comprensión de los temas histológicos? Especifique célula, tejido u órgano'.

\begin{tabular}{|c|c|}
\hline 1. & Barrera hemato-aérea \\
\hline 2. & Conductos \\
\hline 3. & Tejido Nervioso \\
\hline 4. & Modelo como el de las vellosidades \\
\hline 5. & Vasos sanguíneos \\
\hline 6. & De células nerviosas \\
\hline 7. & $\begin{array}{l}\text { Modelos de diferentes células que cumplan } \\
\text { funciones distintas }\end{array}$ \\
\hline 8. & Todos \\
\hline 9. & Nefrona \\
\hline 10. & De todo \\
\hline 11. & Uno con muchos colores \\
\hline 12. & $\begin{array}{l}\text { Las capas del estómago de rumiantes, esófago } \\
\text { y otros }\end{array}$ \\
\hline 13. & Aparato urinario \\
\hline 14. & Alguno relacionado al S.N \\
\hline 15. & Riñón \\
\hline 16. & Todos lo que se puedan hacer \\
\hline 17. & Modelos de células \\
\hline 18. & tejido óseo \\
\hline 19. & $\begin{array}{l}\text { Todos aquellos con diferentes células a } \\
\text { distinguir }\end{array}$ \\
\hline 20. & Sobre todo, para las estructuras complejas \\
\hline 21. & Urinario, medula espinal \\
\hline 22. & Linfonodo \\
\hline 23. & útero \\
\hline 24. & neuroglia, testículo y ovario \\
\hline 25. & córnea \\
\hline 26. & tiroides \\
\hline 27. & músculo sarcómero \\
\hline 28. & tiroides \\
\hline 29. & ojo \\
\hline 30. & Globo ocular \\
\hline 31. & Eje hipotálamo-hipófisis \\
\hline 32. & Tráquea, venas, arterias \\
\hline 33. & Vasos sanguíneos. \\
\hline 34. & glándulas endocrinas \\
\hline 35. & Tejido conjuntivo \\
\hline 36. & Epitelio uterino \\
\hline
\end{tabular}


El trabajo de los docentes en el aula durante las actividades con los estudiantes genera poder aproximarnos a modelos mentales que permiten a un sujeto ir desde la estructura de una célula, tejido u órgano hasta su funcionamiento. Trabajos previos mencionan diversas etapas en la generación de modelos mentales, como ser la representación, visualización, ejecución y contraste con la realidad (Felipe et al., 2005).

Uno de los desafíos es que los estudiantes creen sus propios modelos a partir de la interpretación de imágenes, esto genera que un modelo mental se concrete o sea tangible en una representación tridimensional. Este modo de representación permite expresar relaciones espaciales entre las entidades del modelo previo mental.

Los modelos mentales de los estudiantes pueden contrastarse con los modelos representados, pero impulsan y reafirman la teoría de los modelos mentales donde las representaciones internas que el sujeto utiliza pueden ser revisados recursivamente y modificados ante nuevas confirmaciones descriptivas (Greca y Moreira, 2002).

La creación de modelos $3 \mathrm{D}$ y su uso como recurso didáctico en el aula tiene el potencial para poder discutir y pasar a un plano verbal, según lo observado, generando las instancias de interpretación del modelo en base a sus representaciones previas.

Tal como mencionan investigadores en el campo de la investigación educativa, el uso de visualizaciones se ha vuelto casi omnipresente en la práctica, la enseñanza de las las ciencias (Ford y Minshall, 2019) y en medicina en la clínica (Shafiee y Atala A. 2016). Se define a las visualizaciones como cualquier tipo de representación física diseñada para hacer visible un concepto abstracto. Estos incluyen, entre otros, elementos concretos como fotografías, gráficos en 2D, diagramas, cuadros y modelos en 3D. Dado que los estudiantes sugieren nuevos modelos, se plantea generar nuevas instancias para que los docentes y estudiantes puedan contar con la infraestructura para potenciar la creación de los modelos 3D para el desarrollo de modelos mentales (Uttal y Doherty, 2008).

Asimismo, cabe mencionar que tal como indica Moreira (2017) la educación debe ser dialógica, no hacia el estudiante o del educando hacia el educador, sino del educador con el educando, así como, debe generar más situaciones didácticas que den sentido a los conocimientos para que los estudiantes hablen, participen, pregunten y exterioricen sus significados.

Finalmente, las múltiples sugerencias de creación de modelos 3D realizadas por los estudiantes nos interpelan para seguir mejorando las estrategias didácticas, por ende, la enseñanza y el aprendizaje. A partir de la interdisciplina de saberes pedagógicos, didácticos y tecnológicos se deberán propiciar instancias de aprendizaje profundo mediados por los modelos 3D como recurso didáctico. Coincidimos con Evans (2012) en que la incorporación de la impresión 3D en el aula es una posibilidad real en la educación universitaria en este tiempo y tendrá un lugar creciente en la enseñanza.

A partir de los resultados obtenidos, se podrán considerar nuevas propuestas que incluyan a los estudiantes en el diseño de modelos 3D propiciando el aprendizaje basado en proyectos tal como se menciona en trabajo previos de planificación de estrategias didácticas utilizando modelos 3D (Blázquez et al., 2018), dado que se considera como una herramienta potenciadora de las inteligencias múltiples en la medida que estimula los diferentes canales sensoriales. (Moreno et al., 2016).

En presente estudio determinó que los estudiantes de primer año de Facultad de Veterinaria perciben el uso de modelos 3D a nivel educativo en el aula como un recurso positivo y de interés para su aprendizaje. Estos resultados implican nuevos desafíos para los docentes. En primer lugar, se debería considerar la generación de modelos 3D sugeridos por los estudiantes. Por otra parte, generar el involucramiento de los estudiantes en la creación de nuevos modelos didácticos ayudaría a mejorar la comprensión de temas abordados en clases. $\mathrm{Si}$ consideramos a los modelos 3D como recurso didáctico, esto implica desarrollar contenidos temáticos para la construcción de aprendizajes significativos y formar docentes que estén preparados para trabajar con dichos recursos.

\section{Bibliografía}

Azer SA, Azer S. 2016. 3D Anatomy Models and Impact on Learning: A Review of the Quality of the Literature. Heal. Prof. Educ. 2: 80-98.

Blázquez PJ, Orcos L, Mainz J, Sáez D, Blázquez J. 2018. Propuesta metodológica para la mejora del aprendizaje de los alumnos a través de la utilización de las impresoras 3D como recurso educativo en el aprendizaje basado en proyectos Psicología, Conocimiento y Sociedad, vol. 8, núm. 1, Universidad de la República, Montevideo. Pp. 162-193 DOI:10.26864/pcs.v8.n1.8

Castro M, Ruiz LS, León AT, Fonseca H, íaz M, Umaña W. 2010 Factores académicos en la transición de la primaria a la secundaria: motivación, rendimiento académico y disciplina. Revista Electrónica Actualidades Investigativas en Educación, 10: 1-29.

César-Juárez AA, Olivos-Meza $A$, Landa-Solís $C$, Cárdenas-Soria VH, Silva-Bermúdez PS, Suárez-Ahedo C, Olivos Díaz B, IbarraPonce de León JC. 2018. Novedades en Medicina Uso y aplicación de la tecnología de impresión y bioimpresión 3D en medicina. Revista de la Facultad de Medicina (México) 61: 4351.

Coll RK, France B, Taylor I. 2005. The role of models/and analogies in science education: implications from research. Int. J. Sci. Educ. 27: 183-198. 
Duque JC, Mamián A, Trapasco S. 2012. Uso de las TIC en la práctica pedagógica de los estudiantes de la Licenciatura en Comunicación e Informática Educativas de la Universidad Tecnológica de Pereira. Tesina de grado, Licenciatura en Comunicación e Informática Educativa, Universidad Tecnológica de Pereira, Colombia. 124 pp.

Pérez-Mañanes R, Calvo-Haro J, Arnal-Burró J, Chana-Rodríguez F, Sanz-Ruiz P, Vaquero-Martín J. 2016. Nuestra experiencia con impresión 3D doméstica en Cirugía Ortopédica y Traumatología. Hazlo tú mismo. Rev. Latinoam. Cirugía Ortopédica 1: 47-53.

Evans B. 2012. Practical 3D Printers: The Science and Art of 3D Printing. Appress, New york. 332 pp.

Felipe AE, Gallarreta SC, Merino G. 2005. La modelización en la enseñanza de la biología del desarrollo. Rev. Electrónica Enseñanza de las Ciencias 4: 1-33.

Ford S, Minshall T. (2019). Where and how 3D printing is used in teaching and education. Addit. Manuf. 25: 131-150.

Freeman A, Adams-Becker S, Cummins M, Davis A, Hal Giesinger C. 2017. NMC/CoSN Horizon Report: 2017 K-12 Edition. New Media Consort.

Garas M, Vaccarezza M, Newland G, McVay-Doornbusch K, Hasani J. 2018. 3D-Printed specimens as a valuable tool in anatomy education: A pilot study. Ann. Anat. 219: 57-64.

Gilbert S. 2011. Models-Based Science Teaching. National Science Teachers Association Press, Arlington. 204 pp.

Greca IM, Moreira MA. 2002. Mental, physical, and mathematical models in the teaching and learning of physics. Sci. Educ. 86: 106-121.

Guerrero Armas A. 2009. Los materiales didácticos en el aula. Rev. Digit. para Prof. Enseñanza 5: 1-7.

Juaneda I, Juaneda E, Díaz HO, Peirone A, Massano G, Defago VH. 2018. Impresión tridimensional en doble vía de salida del ventrículo derecho para simplificar la reparación intraventricular. Revista Argentina de Cardiología 86: 209-213.

Mantrana G, Jacobo O, Hartwing D, Giachero V. 2018. Modelos de impresión tridimensional en la planificación preoperatoria y en la enseñanza académica de las fracturas mandibulares. Cirugía Plástica Ibero-Latinoamericana 44: 193-201.

Mejía GP, López MV, Hernández E, Cerano JL. 2019. Diseño de un modelo de evaluación mediante la integración de tecnología inmersiva y a distancia. Educ. Med. 20: 140-145.

Moreira MA. 2017. Aprendizaje significativo como un referente para la organización de la enseñanza. Arch. Ciencias la Educ. 11: e029.

Moreno-Chaustre JJ, Andrade-Sosa HH, García-González JR, Hernández U, Maestre-Góngora GP, López GA. 2014. Modelo de evaluación para valorar el cambio en las prácticas docentes con TIC. Rev. UIS Ingenierías: 13: 7-22.

Moreno NM, Leiva J, López E. 2016. Robótica, modelado 3D y realidad aumentada en educación para el desarrollo de las inteligencias múltiples. Aula de encuentro 18: 158-183.
Shafiee A, Atala A. 2016. Printing Technologies for Medical Applications. Trends Mol. Med. 22: 254-265.

Sieben A, Oparka R, Erolin C. 2017. Histology in 3D: development of an online interactive student resource on epithelium. J. Vis. Commun. Med. 40: 58-65.

Suñol A, Aige V, Morales C, López-Beltran M, Feliu-Pascual AL, Puig J. 2019. Use of Three-dimensional printing models for veterinary medical education: Impact on learning how to identify canine vertebral fractures. J. Vet. Med. Educ. 46: 523532.

Uttal DH, Doherty KO. 2008. Comprehending and Learning from 'Visualizations': A Developmental Perspective. En: Gilbert J.K., Reiner M., Nakhleh M. (eds) Visualization: Theory and Practice in Science Education. Models and Modeling in Science Education, vol 3. Springer, Dordrecht. pp. 53-72.

Vallarta R, Ortíz J, Vázquez M. 2011. Matriz condrocitaria tridimensional (3D) para el dorso nasal: experiencia en rinoplastia cerrada. Cirugía Plástica Ibero-Latinoamericana 37: 331-340.

Vázquez A, Manassero MA. 2008. El declive de las actitudes hacia la ciencia de los estudiantes: Un indicador inquietante para la educación científica. Rev. Eureka Enseñ. Divul. Cien. 5: 272-292. 